\title{
Hybrid Reduced-Order Modeling and Particle-Kalman Filtering for the Health Monitoring of Flexible Structures
}

\section{Giovanni Capellari ${ }^{1}$, Saeed Eftekhar Azam ${ }^{2, *}$ and Stefano Mariani ${ }^{1}$}

1 Politecnico di Milano, Dipartimento di Ingegneria Civile e Ambientale, Piazza L. da Vinci 32, 20133 Milano, Italy; E-Mails: giovanni.capellari@mail.polimi.it (G.C.) ; stefano.mariani@polimi.it (S.M.)

2 University of Thessaly, Department of Mechanical Engineering, Leoforos Athinon, Pedion Areos, 38334 Volos, Greece; E-Mail: eftekhar@mie.uth.gr (S.E.)

* Author to whom correspondence should be addressed; E-Mail: eftekhar@mie.uth.gr (S.E.); Tel.: +30-242-107-4083; Fax: +30-242-107-4050.

Published: 1 June 2014

\begin{abstract}
MEMS-based, surface-mounted structural health monitoring systems were recently proposed to locate possible damage events in lightweight composite structures. To track the structural dynamics induced by the external actions and identify in real-time the inception of drifts from the virgin, or undamaged state, recursive Bayesian filters are here adopted. As the main drawback of any on-line identification method might be linked to the excessive computational costs, two solutions are jointly enforced: an order-reduction of the numerical model used to track the structural behavior, through the proper orthogonal decomposition in its snapshot-based version; an improved particle filtering strategy, which features an extended Kalman updating of each evolving particle before the resampling stage. While the former method alone can reduce the number of effective degrees-offreedom of the structure to a few only (depending on the excitation), the latter allows to track the evolution of damage and also locate it thanks to an intricate formulation. To assess the proposed procedure, the case of a thin plate subject to bending is investigated; it is shown that, when the procedure is fed by measurements gathered by a network of inertial MEMS sensors appropriately deployed over the plate, damage is efficiently and accurately estimated and located.
\end{abstract}

Keywords: structural health monitoring; reduced-order modeling; proper orthogonal decomposition; particle-Kalman filtering; inertial MEMS 


\section{Introduction}

The aim of this work is the development of an online damage identification method, or structural health monitoring strategy based on recursive Bayesian filters. Its main feature is the fast, robust and unbiased estimation of damage indexes associated to any given structure or, in other words, the estimation of the local stiffness of the system, by using partial observations of the whole structural state. This goal is attained by allowing for an effective model order reduction technique, to guarantee a reduced computational cost, and by coupling the procedure with a commercial finite element (FE) code. Hence, the strategy can be applied to a large variety of problems, featuring a wide range of FE formulations.

As already pointed out here above, one of the main disadvantages of any identification technique based on recursive Bayesian filters is the entailed high computational burden. Two remedies are here adopted to mitigate such issue: a model order reduction, and an improved filtering strategy. The model order reduction is achieved by adopting a Galerkin-based projection of the original full model into a sub-space spanned by the so-called proper orthogonal modes (POMs), computed with the proper orthogonal decomposition (POD) in its snapshot-based version [1-3]. The formulation is organized in such a way that it results independent of the FE model used to discretize the structure and of its implementation. Hence, only some damage indexes affecting the original stiffness of the structure need to be handled. A dual estimation of the reduced order state and of damage parameters, together with an online update of the subspace, is based on a particle filter enhanced through the use of a further (extended) Kalman filter to move the particles before the resampling stage [2-4]. This intricate formulation allows tracking both the damage parameters and the dynamic evolution of the partially observed or hidden state of the system.

In order to assess the capability of the identification procedure, a damaged thin plate case is considered. It is shown that the filter is able to identify and locate the damage even using a very reduced order of the system. The premises for feasibility of the presented method relies on recent developments in smart embedded data acquisition systems and MEMS (micro electro-mechanical systems) type accelerometers, that allow to collect vibration data for lightweight and small structures without altering their response [5].

Methods similar to those adopted herein for the dual estimation and subspace update were recently developed and successfully applied to shear-type buildings [1-3]. The current study represents an evolution of previous works, as it proves to be able to locate and estimate (almost) in real time a structural damage via a few vibration measurements only.

\section{Problem formulation}

To estimate the damage indexes and the current state of the system altogether, we make recourse to recursive Bayesian filters. An extended Kalman particle filter is used for joint state and parameter estimation, and a further Kalman filter is used to update the subspace provided by the POD.

Likewise all recursive Bayesian inference algorithms, the procedure is started from an initial guess. Then, within each time interval $\left[t_{k-1} t_{k}\right]$ it can be assumed that the estimations of state, parameters 
and subspace of the system are initially available; the joint state and parameter estimation is next updated exploiting the following state space formulation:

$$
\begin{aligned}
\boldsymbol{x}_{r, k} & =\boldsymbol{f}_{r, k}\left(\boldsymbol{x}_{r, k-1}\right)+\boldsymbol{v}_{k} \\
\boldsymbol{y}_{k} & =\boldsymbol{H}\left[\begin{array}{llll}
\boldsymbol{\Phi}_{l, k} & & & \\
& \boldsymbol{\Phi}_{l, k} & & \\
& & \boldsymbol{\Phi}_{l, k} & \\
& & & \mathbf{0}
\end{array}\right] \boldsymbol{x}_{r, k}+\boldsymbol{w}_{k}
\end{aligned}
$$

where: $\boldsymbol{x}_{r, k}=\left[\begin{array}{ll}\boldsymbol{z}_{r, k} & \boldsymbol{\vartheta}_{k}\end{array}\right]^{T}$ is the extended state of the system; $\boldsymbol{z}_{r, k}$ is the current state furnished by the reduced order model, ROM (hence, subscript $r$ stands for the reduced state); $\boldsymbol{\vartheta}_{k}$ collects the sought model parameters, namely the damage indexes $d_{i}, i=1, \ldots, N_{d}$, that affect the structural stiffness matrix $\boldsymbol{K}_{r, k}\left(d_{i}\right)$ of the reduced system; $\boldsymbol{v}_{k}$ is a zero mean, white Gaussian noise that represents the uncertainties in the process equation; $\boldsymbol{y}_{k}$ is the partially measured state of the system; $\boldsymbol{w}_{k}$ is the relevant measurement uncertainty vector; $\boldsymbol{H}$ is a Boolean matrix that links the observation vector to the current state; $\boldsymbol{\Phi}_{l, k}$ denotes the matrix that included the first $l$ POMs of the system [1-3], $l$ being the order of the reduced model.

Along with Eqs. (1) and (2), an additional one has to be considered in order to allow a time variation and update of the reduced order subspace via $\boldsymbol{\Phi}_{l}$ :

$$
\boldsymbol{\Phi}_{l, k}=\boldsymbol{\Phi}_{l, k-1}+\boldsymbol{v}
$$

where $\boldsymbol{v}$ denotes a fictitious zero mean, white Gaussian noise with associated covariance $\boldsymbol{\Upsilon}$. Like the other parameters of the proposed intricate formulation, $\mathbf{Y}$ needs to be tuned to obtain unbiased estimates of the subspace vectors. Table 1 provides the general outline of the method here proposed to estimate the damage indexes, track the evolution of the full state of the system and update the subspace spanned by the POMs of the structure.

Table 1. General scheme of the proposed damage identification method.

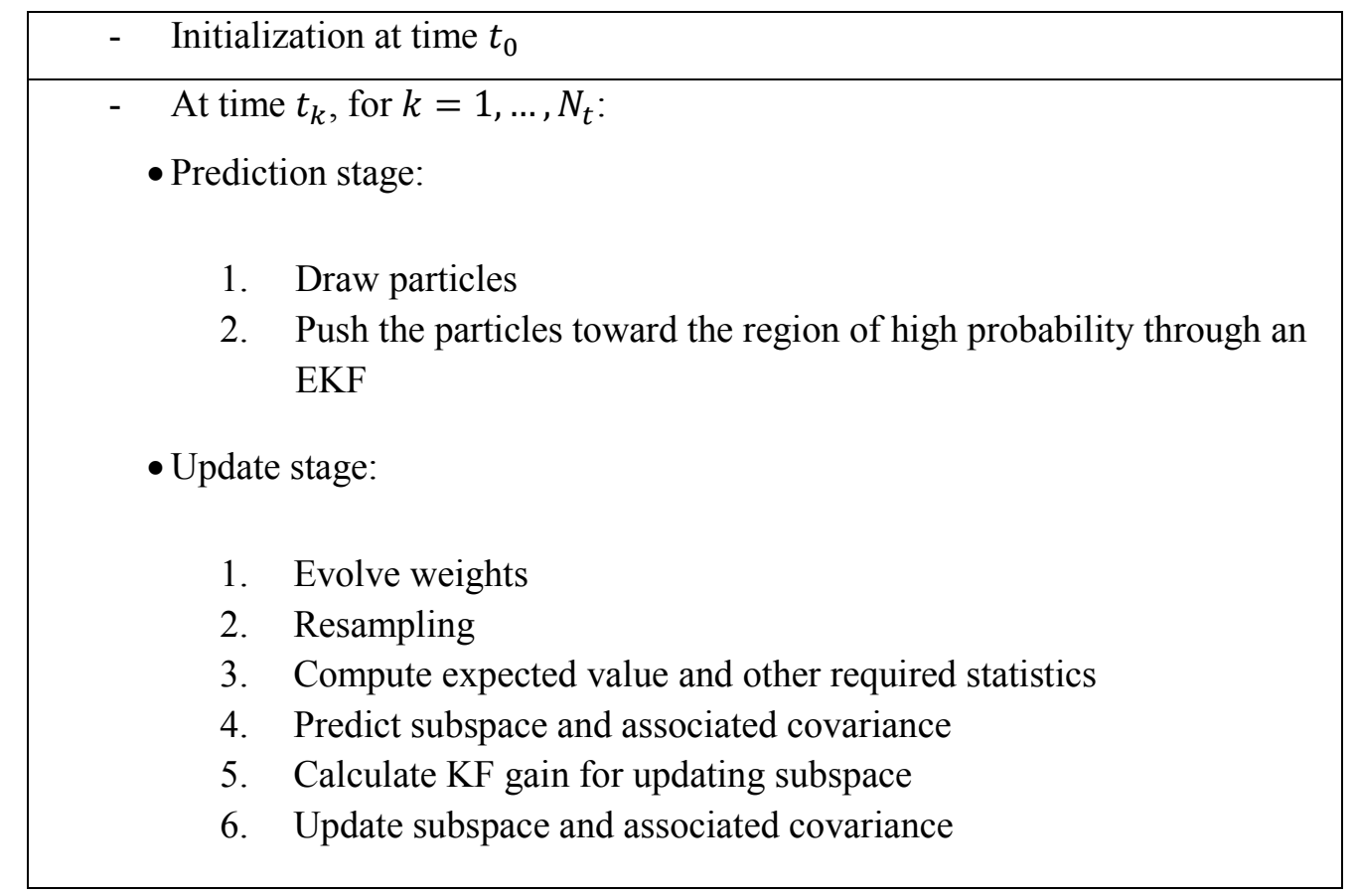




\section{Results and Discussion}

To assess the capability and performances of the proposed approach, a benchmark test is considered. A square plate, whose width is $200 \mathrm{~mm}$ and thickness is $5 \mathrm{~mm}$, is assumed to be made of Aluminum 6061-T6 (with Young's modulus $E=68.9 \mathrm{MPa}$ and density $\rho=2.5 \cdot 10^{3} \mathrm{~kg} / \mathrm{m}^{3}$ ). A point load is applied at the mid-span, perpendicularly to the reference mid-plane; the maximum value of the load has been tuned in order for the material to always behave elastically. As far as boundary conditions are concerned, the plate is simply supported at the four corners. The plate has been modeled with the commercial FE code Abaqus, using the S4R general-purpose shell elements to take into account transverse shear deformations. Figure 1 depicts the problem boundary and loading conditions, along with the (artificial) subdivisions of the plate into four zones, or regions featuring their own damage indexes $d_{i}, i=1, \ldots, 4$. Needless to say, such decomposition into zones can be totally independent of the background mesh adopted to space discretize the structure.

Figure 1. Benchmark text: boundary conditions, load and zone numbering.

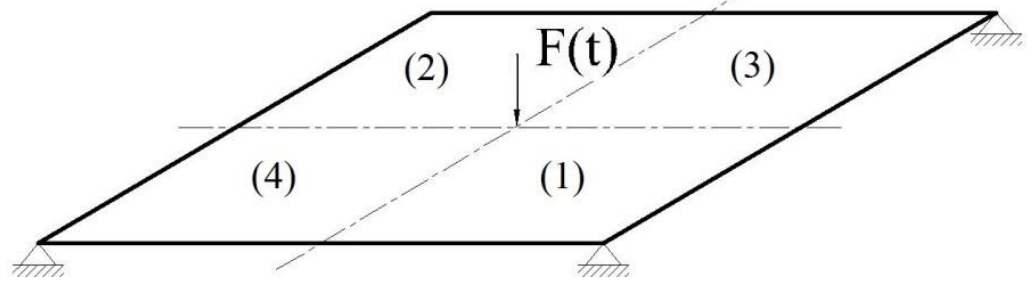

To specifically assess the performance of the damage detection scheme, two configurations have been considered, both characterized by a non-zero damage $d_{1}$ only in the first region: a former one is representative of a time-invariant state of the plate, where $d_{1}=0.5$ throughout the whole analysis (namely from $t=0$ ); a latter one is instead representative of a time-varying plate health, where $d_{1}=0.5$ up to a specific time instant beyond which it instantaneously grows to $d_{1}=0.7$. Results reported hereafter can be shown to be rather invariant with the background space discretization.

As for the time-invariant case, method performances are evaluated in Figure 2 as a function of the number of POMs, i.e. of the number of degrees of freedom retained in the ROM, in terms of estimations of the damage indexes starting from a trivial null initial guess. It must be mentioned that the observation data have been collected in this analysis during a pseudo-experimental test, wherein a full order simulation of the plate dynamics induced by a sinusoidally varying load has been run. It is clearly shown that a fast and well accurate estimate of $d_{1}$ has been obtained by handling a reduced order model that features only 3 POMs. Figure 2(d) also shows the Euclidean norm of the relative estimation error of the damage indexes at varying number of POMs: it emerges that going beyond $l=3$ would increase the computational burden without enhancing much the accuracy of the outcomes.

As for the time-varying case, Figure 3(a) shows the results obtained when the subspace update strategy is purposely dropped in the joint state and parameter estimation, whereas Figure 3(b) gathers the same results obtained when the aforementioned subspace update is plugged in. It is seen that, without the proposed online update of the subspace matrix the scheme fails to track the evolution of the damage index $d_{1}$; this detrimentally affects also the estimates of the other non-evolving indexes. 
Figure 2. Benchmark test with time-invariant damage state: (a) damage indexes $d_{i}$ identified with one POM retained in the ROM; (b) damage indexes $d_{i}$ identified with two POMs; (c) damage indexes $d_{i}$ identified with three POMs; (d) relative error of damage index estimation at varying order $l$ of the ROM.

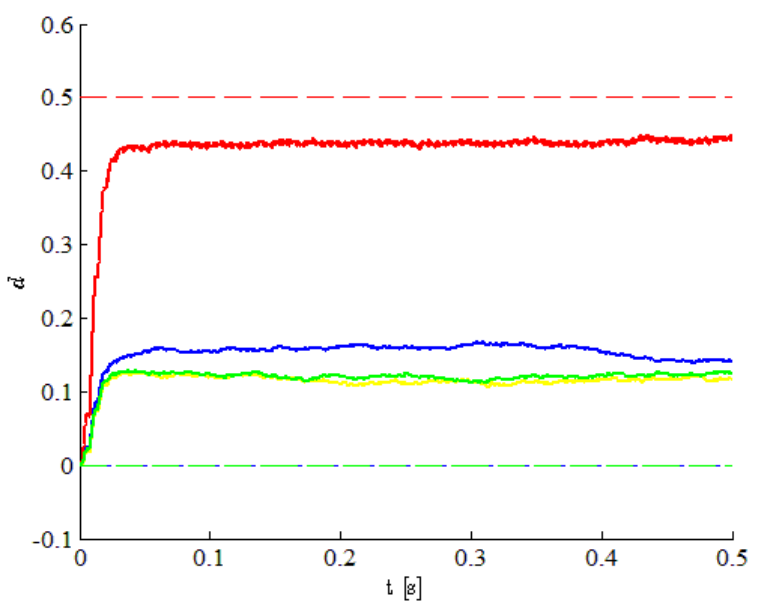

(a)

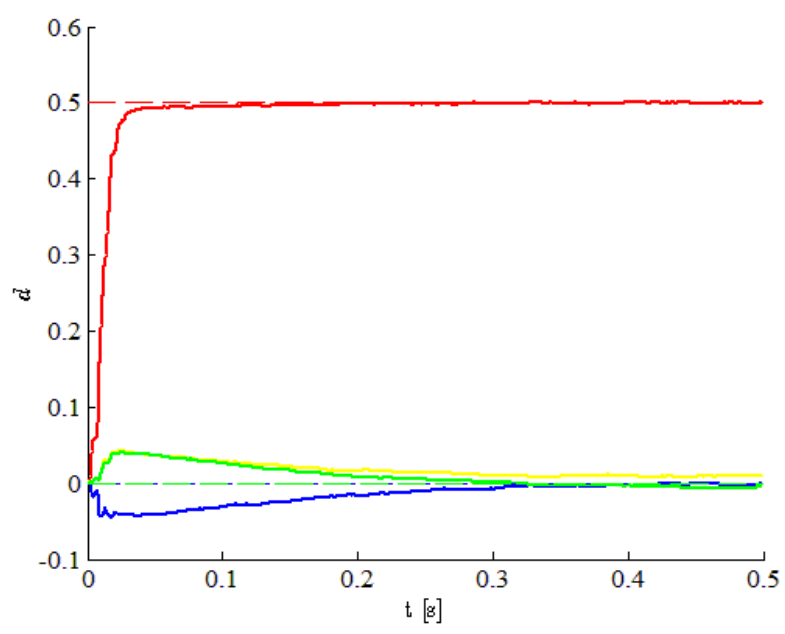

(c)

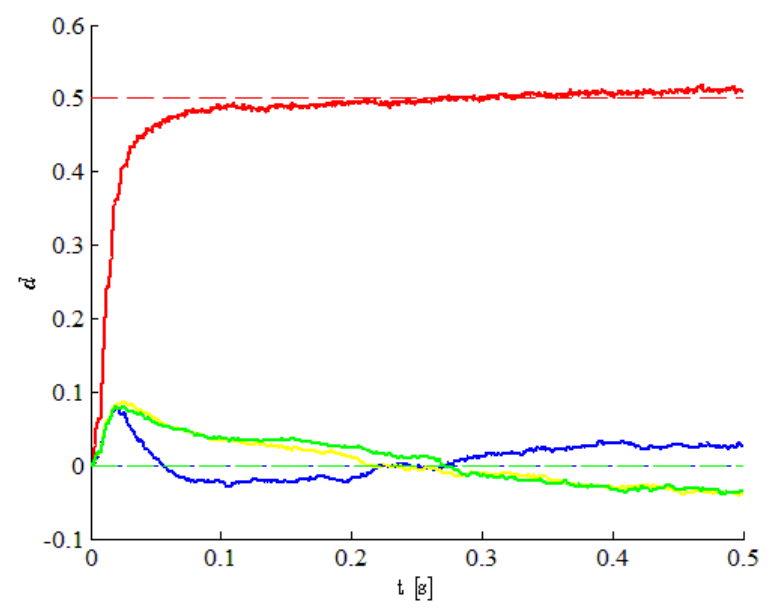

(b)

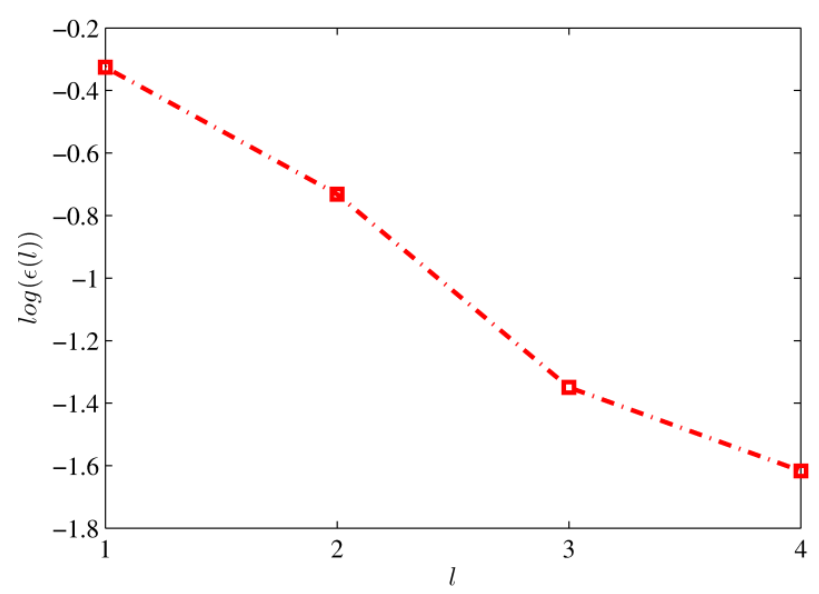

(d)

\section{Conclusions}

In this work, a new online damage identification method based on recursive Bayesian filters has been presented, and its application to a thin plate case has been shown. The method is based on an improved particle filtering strategy, wherein each generated particle is updated using also an extended Kalman filter, and on an order reduction of the numerical model used to track the structural behavior. As for this last stage, the proper orthogonal decomposition in its snapshot-based version has been adopted. The main innovation with respect to previous works concerns the possibility to locate and accurately estimate the damage parameters associated to the local reduction of the structural stiffness. Moreover, although this aspect has not been discussed here in details, the method has been coupled with a commercial finite element code, to allow the algorithm to perform as a general estimation strategy. 
Figure 3. Benchmark test with time-evolving damage state: (a) damage indexes $d_{i}$ identified without POM update; (b) damage indexes $d_{i}$ identified with POM update.

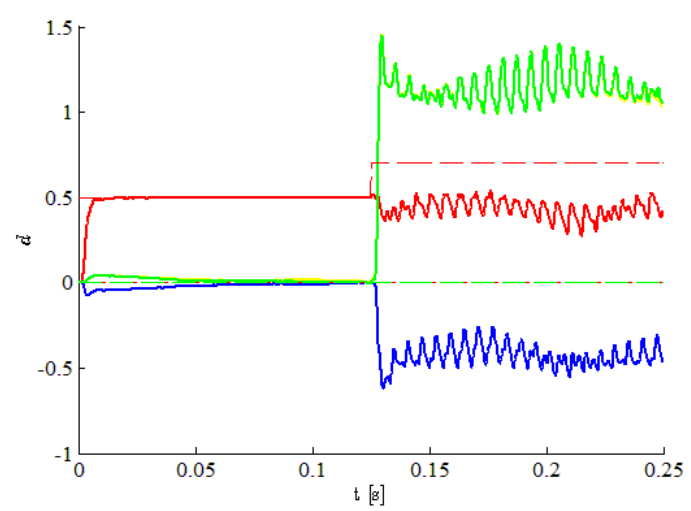

(a)

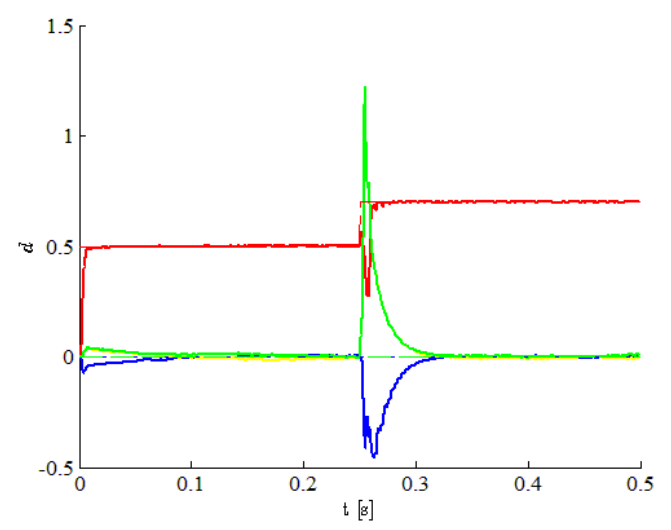

(b)

\section{Acknowledgments}

A partial financial support from Fondazione Cariplo through project Safer Helmets is gratefully acknowledged.

\section{References and Notes}

[1] Eftekhar Azam, S.; Mariani, S. Investigation of computational and accuracy issues in POD-based reduced order modeling of dynamic structural systems. Engineering Structures 2013, 54, 150167.

[2] Eftekhar Azam, S. Dual estimation and reduced order modelling of damaging structures. $\mathrm{PhD}$ Thesis, Politecnico di Milano, 2012.

[3] Eftekhar Azam, S. Online Damage Detection in Structural Systems. Springer, 2014.

[4] Eftekhar Azam, S.; Mariani, S. Dual estimation of partially observed nonlinear structural systems: A particle filter approach. Mechanics Research Communications 2012, 46, 54-61.

[5] Mariani, S.; Corigliano, A.; Caimmi, F.; Bruggi, M.; Bendiscioli, P.; De Fazio, M. MEMS-based surface mounted health monitoring system for composite laminates. Microelectronics Journal 2013, 44, 598-605.

(C) 2014 by the authors; licensee MDPI, Basel, Switzerland. This article is an open access article distributed under the terms and conditions of the Creative Commons Attribution license (http://creativecommons.org/licenses/by/3.0/). 